\title{
Optical and Near-IR Monitoring of Symbiotic Binary Systems
}

\author{
Joanna Mikolajewska \\ N. Copernicus Astronomical Center, Bartycka 18, 00716 Warsaw, \\ Poland, e-mail: mikolaj@camk.edu.pl
}

\begin{abstract}
Symbiotic stars are long-period interacting binary systems in which an evolved red giant star transfers material to its much hotter compact companion. Such a composition places them among the most variable stars. In addition to periodic variations due to the binary motion, they often show irregular changes due to nova-like eruptions of the hot component. In some systems the cool giant is a pulsating Mira-type star usually surrounded by a variable dust shell. Here, I present results of optical and IR monitoring of symbiotic systems as well as future prospects for such studies.
\end{abstract}

\section{Introduction}

Most stars in the Universe are binaries. Among them, symbiotic stars are interacting binaries in which an evolved giant transfers material to a hot and compact companion. In a typical configuration, a symbiotic binary contains an M III giant and a white dwarf accreting material lost in the cool giant wind. The wind is ionised by the hotter of the binary components giving rise to symbiotic nebula (cf. Mikołajewska 1997).

Based on the near-IR colours, two distinct classes of symbiotic stars have been defined (Allen 1983): the S-type (stellar) with normal red giants, and the D-type (dusty) with Mira primaries surrounded by a warm dust shell. The distinction between the $S$ and $D$ types seems to be one of orbital separation: the binary must have enough space for the red giant, and yet allow it to transfer sufficient mass to its companion. In fact, all symbiotic systems with known orbital periods - of the order of a few years - belong to the S-type, while the orbital periods for the D-type systems are generally not known, probably because they are longer than periods covered by existing observations (cf. Belczyński et al. 2000).

Symbiotic stars are thus interacting binaries with the longest orbital periods and the largest component separations, and their study is essential to understand the evolution and interactions of detached and semi-detached binary stars. They are also among the brightest (intrinsically) stars, which makes them excellent observational targets both in our Galaxy as well as in nearby galaxies even for relatively small telescopes.

In the following, I will present results of optical and infrared monitoring of symbiotic stars as well as future prospects for such studies. 


\section{Variable Phenomena in Symbiotic Stars}

The composition of a typical symbiotic binary, specifically the presence of an evolved giant and its accreting companion, places symbiotic stars among the most variable stars. They can fluctuate in several different ways, which can be revealed and studied by patient monitoring of their light curves and radial velocity changes. Namely, binary motion can be manifested by eclipses of the hot component by the giant, by modulation of the giant's light due to reflection effect (with orbital period) and due to tidal distortion (with $P_{\text {orb }} / 2$ ), as well as radial velocity changes. The cool giant can also show intrinsic variability, in particular, radial pulsations (all D-type and some S-type systems) and semiregular variations (S-type) with timescales on the order of months and years as well as long-term light variations due to variable obscuration by circumstellar dust (most D-type systems), solar-type cycles, spots, etc. The effects of mass accretion onto the hot component also involve different variable phenomena. The hot component in the vast majority of symbiotic systems seems in fact to be a luminous $\left(\sim 1000 \mathrm{~L}_{\odot}\right)$ and hot $\left(\sim 10^{5} \mathrm{~K}\right)$ white dwarf powered by thermonuclear burning of the material accreted from its companion's wind. Depending on the accretion rate, these systems can be either in a steady burning configuration or undergo hydrogen shell flashes. In many cases such flashes can last for decades due to the low mass of the white dwarf (Mikołajewska 1997). In addition, the hot components in many systems show activity with timescales of a few years which cannot be simply accounted for by the thermonuclear models. A possible and promising explanation involves fluctuations in mass transfer and/or accretion instabilities.

Below, I present examples of light curves for well-studied, though not yet completely understood, symbiotic binaries: RX Pup, CI Cyg and CH Cyg, which are representative for the wealth of variable phenomena observed in these systems.

\subsection{RX Puppis: a Possible Recurrent Nova with a Symbiotic Mira Companion}

RX Pup is a symbiotic binary composed of a long-period Mira variable pulsating with $P \approx 578$ days, surrounded by a thick dust shell, and a hot $\sim 0.8 \mathrm{M}_{\odot}$, white dwarf companion. The binary separation could be as large as $a \geq 50$ a.u. (corresponding to $P_{\text {orb }} \geq 200 \mathrm{yr}$ ) as suggested by the permanent presence of a dust shell around the Mira component (Mikolajewska et al. 1999). In particular, the Mira is never stripped of its dust envelope, and even during relatively unobscured phases the star resembles the high-mass loss galactic Miras with thick dust shells. In general, the binary component separations in D-type systems must be larger than the dust formation radius. Assuming a typical dust formation radius of $z 5 \times R_{\text {Mira }}$, and a Mira radius of $R_{\text {Mira }} \sim 1-3$ au (e.g. Haniff, Scholz \& Tuthill 1995), the minimum binary separation is $a \gtrsim 20 \mathrm{au}$, and the corresponding binary period is $P_{\text {orb }} \gtrsim 50 \mathrm{yr}$, for any D-type system.

Recent analysis of multifrequency observations shows that most, if not all, photometric and spectroscopic activity of RX Pup in the UV, optical and radio range is due to activity of the hot component, while the Mira variable and its circumstellar environment is responsible for practically all changes in the infrared 


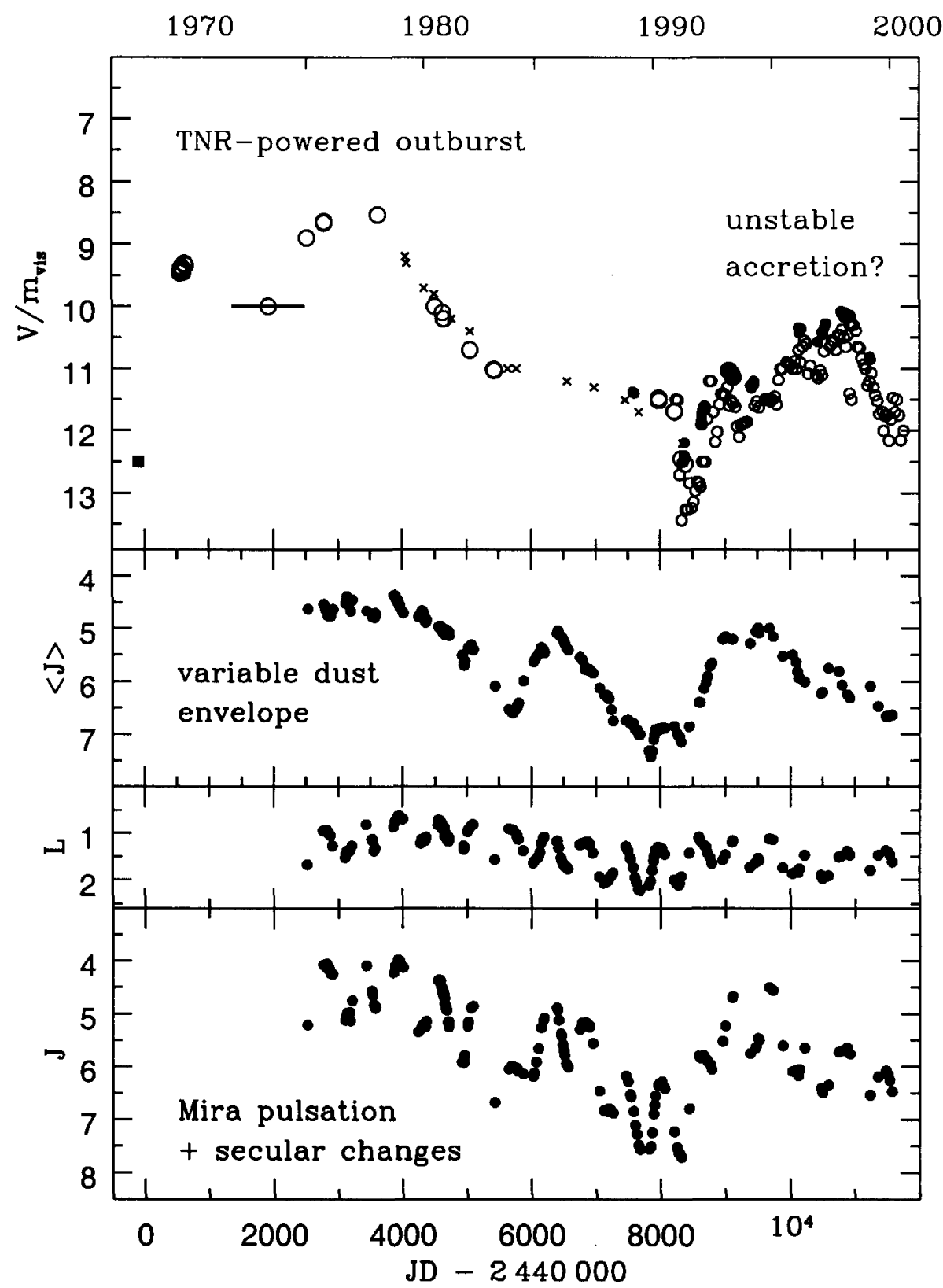

Figure 1. Optical and IR light curves of RX Pup from Mikołajewska et al. (1999). In the $V / m_{\text {vis }}$ light curve, small open circles represent observations from RASNZ; large open circles and dots published V magnitudes; crosses FES magnitudes. The optical light is dominated by the hot component activity whereas the IR light curves are dominated by the Mira pulsation ard variable dust obscuration of the Mira by circumstellar dust. 
range (Mikołajewska et al. 1999, and Fig. 1). In particular, RX Pup underwent a nova-like eruption during the last three decades. The hot component contracted in radius at nearly constant luminosity from 1975 to 1986 , and was the source of strong stellar wind, which prevented it from accreting material lost in the Mira wind. Around 1988/9, the hot component turned over the HR diagram and by 1991 its luminosity had faded by a factor of $\sim 30$ with respect to the maximum plateau value (see the very deep minimum in the visual light curve in Fig.1) and the hot wind had practically ceased. By 1995 the nova remnant started to accrete material from the wind, as indicated by a general increase of the optical flux. The earliest observational records from the 1890s suggest that another nova-like eruption of RX Pup occurred around 1894.

The near-IR light curves show significant long-term variations in addition to the Mira pulsation (Fig. 1). The long-term changes are best visible in the $\langle J\rangle$ light curve after removal of the Mira pulsation (middle panel in Fig. 1). Mikolajewska et al. (1999) have found large changes in the reddening towards the Mira accompanied by fading of the near IR flux. However, the reddening towards the hot component and the emission line regions remained practically constant and was generally less than that towards the Mira. These changes do not seem to be related to the orbital configuration nor to the hot component activity. Similar dust obscuration events seem to occur in many well covered symbiotic Miras (e.g. Whitelock 1998), as well as in single Miras (e.g. Mattei 1997, Whitelock 1998), and they are best explained as intrinsic changes in the circumstellar environment of the Mira variable, possibly due to intensive and variable mass loss. The last increase in extinction towards the Mira in RX Pup has been accompanied by large changes in the degree of polarization in the optical and red spectral ranges. This confirms that these long-term variations are driven by changes in the properties of the dust grains, such as variable quantity of dust and variable particle size distribution, due to dust grain formation and growth (Mikołajewska et al. 2001).

\subsection{Cygni: a Tidally Distorted Giant with a Disc-accreting Sec- ondary}

Although most symbiotic binaries seem to interact by wind-driven mass loss, a few of them may contain a Roche-lobe filling giant. They also show activity with time scales on the order of years that can be related to the presence of accretion discs. Among them, CI Cyg is one of the best studied. Kenyon et al. (1991) demonstrated that it consists of an M5 II asymptotic branch giant, $M_{\mathrm{g}} \sim 1.5 \mathrm{M}_{\odot}$, and a $\sim 0.5 \mathrm{M}_{\odot}$ hot companion separated by $2.2 \mathrm{AU}$. They also argued that the hot companion is a disc-accreting main sequence star. However, quiescent IUE data from the early 1990s can also be accounted for by a hot and luminous stellar source powered by thermonuclear burning which makes the case for CI Cyg as an accreting MS star less clear.

The outburst light curve of CI Cyg ( Fig. 2) in addition to deep eclipses of the hot component by the red giant shows a $0.5-1.0$ mag oscillations with a period of $\sim 0.9 P_{\text {orb }}$. Some other S-type systems show similar secondary periodicities best visible in their outburst light curves, in all cases $10-15 \%$ shorter than the orbital periods. The nature of this secondary periodicity is unknown. Recently, Galis et al. (1999) suggested that in the case of AG Dra, it is due 

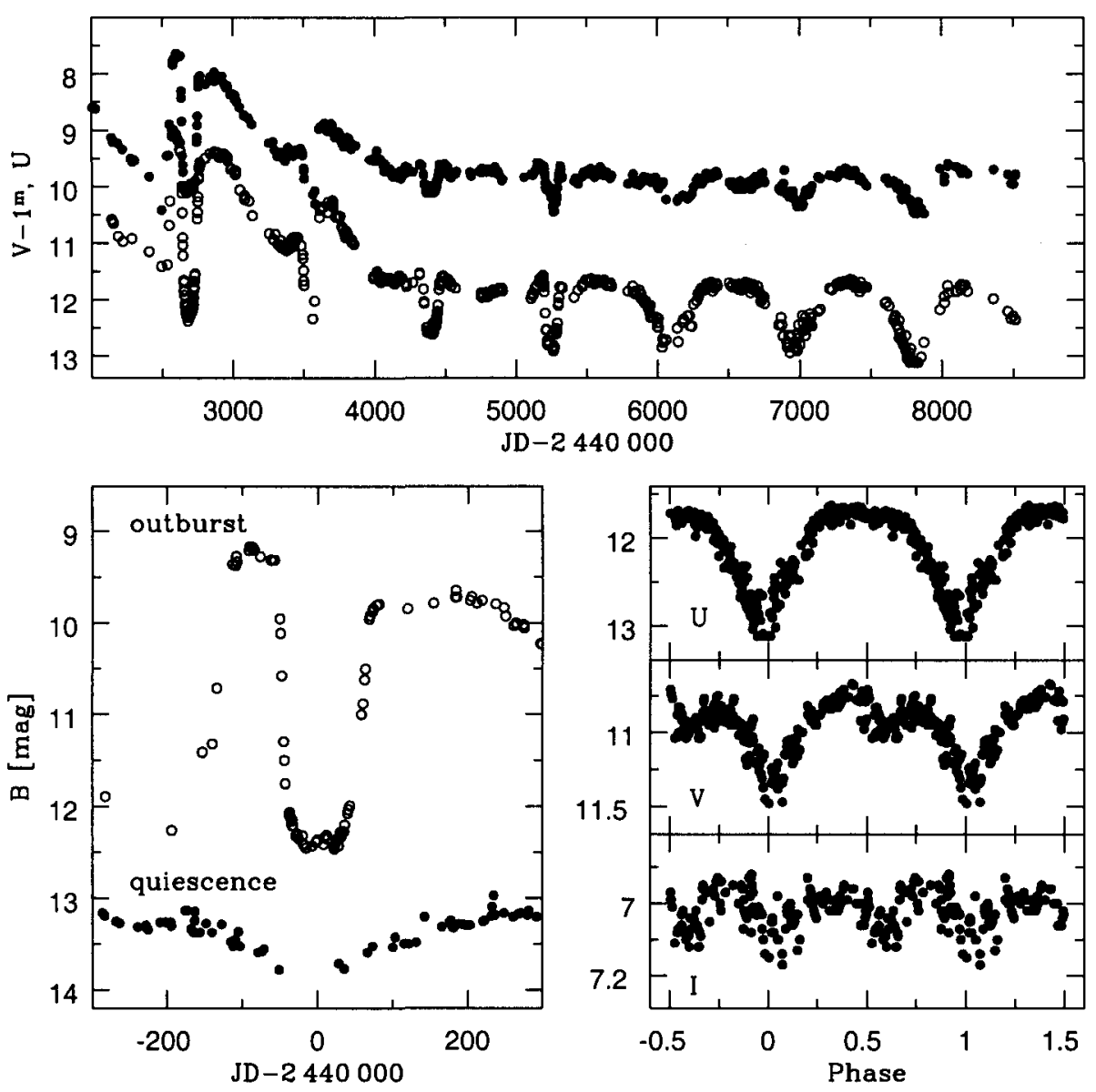

Figure 2. Optical and near infrared light curves of CI Cyg. The $U B V I$ data are from Belyakina $(1979,1984,1991,1992)$, Khudyakova (1989) and Meuninger (1981). Deep eclipses and the large outburst which started in 1975 are the most prominent features of the optical light curves (upper panel). During the outburst and its decline eclipses were narrow with well-defined eclipse contacts whereas the quiescent light curves show very broad minima and almost continuous sinusoidal variation (left panel). The ellipsoidal variability of the red giant is visible only in quiescent visual and red light curves (right panel). 
to radial pulsations of the giant, and the outbursts are driven by resonances between the pulsations and binary motion. On the other hand, the secondary periodicities are best visible in the optical light where the contribution from the giant, especially during the outburst, is very low or negligible. There is also a striking similarity between these variations and the superhumps of the SU UMa class of CVs which may indicate that they are rather related to the presence of accretion discs (Mikołajewska \& Kenyon 1992).

During the outburst and its decline, eclipses in the $U B V$ continua and optical H II, HeI and HeII emission lines were narrow with well-defined eclipse contacts whereas at quiescence very broad minima and continuous nearly sinusoidal changes were observed. In addition, the quiescent $V R I$ light curves show a modulation with $P_{\text {orb }} / 2$ as expected for an ellipsoidal light curve. The amplitude of this modulation, $\Delta I \sim 0.15$ is consistent with the system inclination, $i \sim 73^{\circ}$, and the mass ratio, $M_{\mathrm{g}} / M_{\mathrm{h}} \sim 3$ derived by Kenyon et al. (1991). The transition from narrow eclipses to sinusoidal variations was accompanied by large spectral changes and appearance of a radio emission with a spectral distribution that cannot be simply accounted for by any of the popular models for symbiotic stars (Mikołajewska \& Ivison 2001).

\subsection{CH Cygni: Triple or Binary System with a Magnetic White Dwarf}

The record for the complexity of variable phenomena found in a single symbiotic object may be held by $\mathrm{CH}$ Cyg, the symbiotic system with the longest $\left(P_{\text {orb }} \sim 15.5 \mathrm{yr}\right)$ measured orbital period (Mikołajewski, Tomov, \& Mikolajewska 1987; Hinkle et al. 1993), whoose light curves are presented in Fig. 3. Both the light curves and the radial velocity curves show multiple periodicities: a $\sim 100^{\mathrm{d}}$ photometric period, best visible in the $V R I$ light curves, which has been attributed to radial pulsation of the giant (Mikołajewski, Mikolajewska, \& Khudyakova 1992), while the nature of the secondary period of $\sim 756^{\mathrm{d}}$ also present in the radial velocity curve is not clear (Hinkle et al. 1993; Munari et al. 1996). There is a controversy about whether the system is triple or binary, and whether the symbiotic pair is the inner binary or the white dwarf is on the longer orbit. The near-IR light curves also show long-term variations similar to the dust obscuration phenomena found in symbiotic Miras (cf. Munari et al. 1996).

The hot component also shows very spectacular activity. In particular, we deal with irregular outbursts accompanied by fast, massive outflows and jets, rapid brightness variations with a time scale on the order of minutes, and other peculiarities which cannot be explained in the frame of the classical models proposed for symbiotic stars. Mikołajewski et al. (1990) proposed that this peculiar activity is powered by unstable accretion onto a magnetic white dwarf secondary.

\section{Present State-of-the-art and Future Prospects}

The recently published catalogue of symbiotic stars includes 188 symbiotic stars as well as 30 objects suspected of being symbiotic (Belczyński et al. 2000), Among them, 173 are in our Galaxy, 14 in the Magellanic Clouds and 1 in 

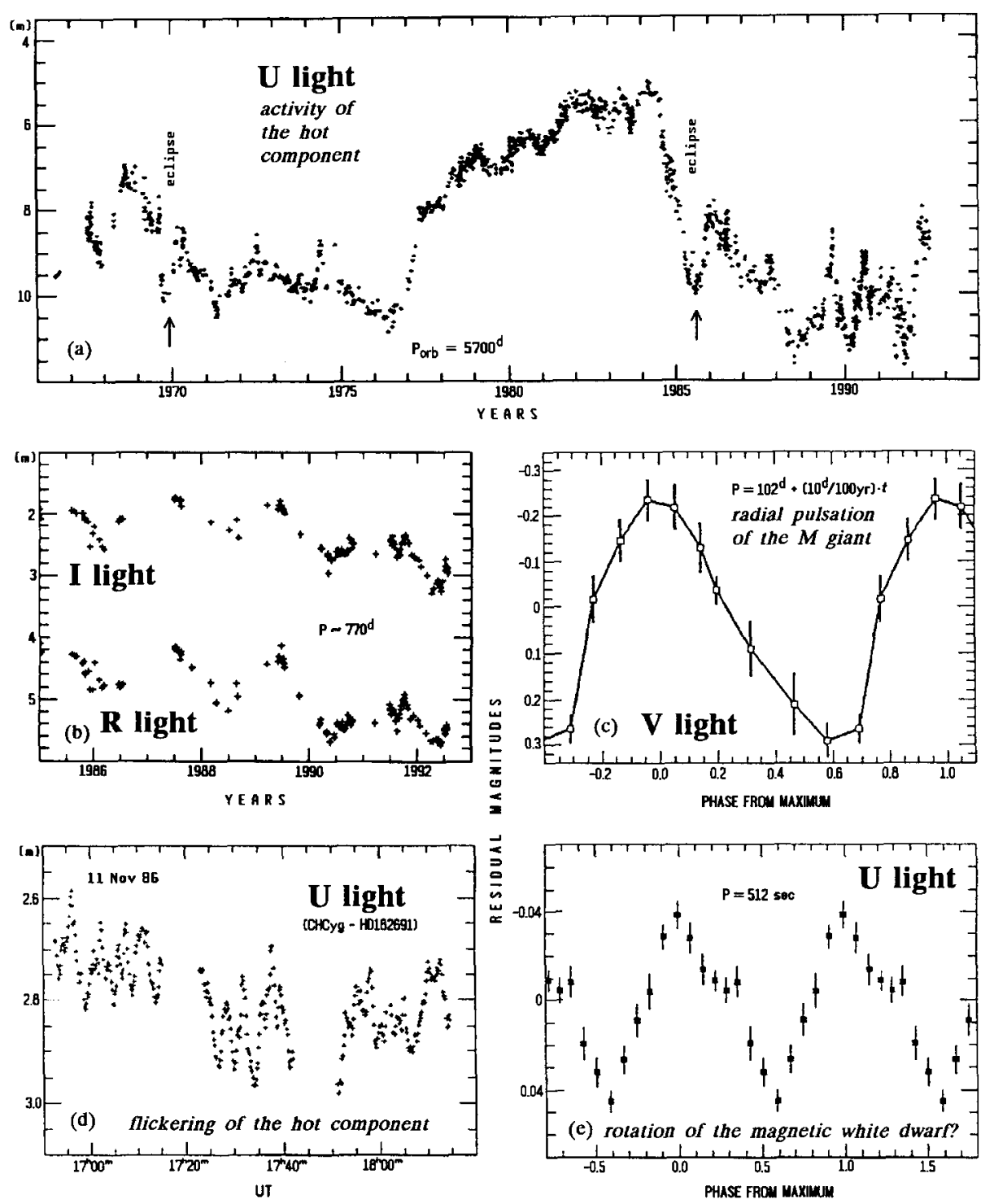

Figure 3. Variable phenomena in $\mathrm{CH}$ Cyg: (a) $\mathrm{U}$ light curve from the recent series of outbursts. Arrows indicate times of eclipses of the hot component. (b) $\mathrm{R}$ and I light curves of the $M$ giant (c) Mean light curve for residuals of the $V$ magnitude binned and folded with the $102^{\mathrm{d}}$-pulsation period of the $\mathrm{M}$ giant (Mikolajewski et al. 1992). (d) Rapid variability in U light. (e) Residual normal points of the data plotted in panel (d) folded with the 512-sec period (Mikolajewski et al. 1990). 
Draco-1. They are excellent targets for small telescopes, especially for longterm monitoring of their complex photometric and spectroscopic variability. Although we have $\sim 120$ S-type symbiotic systems with $V \lesssim 15^{\mathrm{m}}$, photometric orbital periods have been measured for only 30 objects ( 18 of then are eclipsing). Twentyone systems have also known spectroscopic orbits and for 8 of them mass ratios have been also estimated. Ellipsoidal light variations, characteristic of tidally distorted stars, have rarely been observed. Thus far, only four systems, T CrB, CI Cyg, BD-21 3873 , and possibly EG And, seem to show such changes. The general absence of the tidally distorted giant in symbiotic binaries may however be due to the lack of systematic searches for the ellipsoidal variations in the red and near-IR range, where the cool giants dominate the continuum light. On the other hand, tidal interactions are certainly important in symbiotic systems as suggested by the practically circular orbits of most $(\sim 80$ $\%$ ) systems with known orbital solutions, and specifically of all those showing the multiple CI Cyg-type outburst activity. We do not know the orbital period for any of the extragalactic symbiotic stars, although 8 of them belong to the S-type, and with $V \sim 15-17 \mathrm{mag}$, are bright enough for optical monitoring even with a relatively small telescope.

Similarly, among 33 galactic D-type systems $\left(K \leqslant 8^{\mathrm{m}}\right)$, pulsation periods have been observed - and thus the Mira presence confirmed - for only 12 systems. Pulsation periods are also unknown for the few extragalactic D-type systems $\left(K \sim 10-13^{\mathrm{m}}\right)$.

Optical and near-IR monitoring of symbiotic stars is essential not only to the understanding of variable phenomena in symbiotic stars and more generally longperiod interacting binaries, but also to studying such phenomena in several other astrophysical environments (giant stars, planetary nebulae, novae, supernovae, supersoft X-ray sources, hot stars and even AGNs).

Studies of the symbiotic Miras are important for understanding the evolution and interaction of detached low-mass binaries. For example, there is ample observational evidence for systematic differences between the symbiotic Miras and average single galactic Miras. In particular, their average pulsation periods are longer, the colours redder and the mass-loss rates higher than typical periods, colours, and mass-loss rates for single Miras (cf. Mikolajewska 1999). It is interesting which and how these differences are related to the binary nature of symbiotic Miras. The symbiotic Miras are often associated with extended radio and/or optically resolved nebulae. These nebulae have usually very complex structure, often with bipolar lobes and jet-like features.

There are also many important questions posed by the active S-type systems. What powers the multiple outburst activity in CI Cyg and other similar systems? How many of these contain tidally distorted giants? What is the nature of the secondary periodicity, $\varsigma 0.8-0.9 P_{\text {orb }}$, visible at outburst in some of them? Can the secondary periodicities be considered as evidence for the presence of an accretion disc? Such periodicities have not been found in any symbiotic nova during either optical maximum or constant luminosity phase (the plateau portion of white dwarf cooling tracks), including the best studied case - AG Peg (Kenyon et al. 1993), and their presence indicates that the outbursts in CI Cyg and other similar systems are not powered by thermonuclear reactions. The timescales and relative amplitudes for these eruptions are very similar to 
the timescales and amplitudes of the hot component luminosity variations (high and low states) in symbiotic recurrent novae (e.g. T $\mathrm{CrB}$, RS Oph, RX Pup) between their nova eruptions (Anupama \& Mikolajewska 1999; Mikolajewska et al. 1999) and in other accretion-powered systems (CH Cyg, Mira A+B). It is possible that the main difference between CI Cyg, Z And, AX Per, and other related symbiotic systems with multiple eruption activity and the activity of accretion-powered systems (symbiotic recurrent novae and $\mathrm{CH} \mathrm{Cyg}$ ) is that the hot component in the former burns more or less stably the accreted hydrogen whereas not in the latter. Systematic optical and near infrared monitoring with small telescopes can provide an answer to these and many other questions.

Finally, in 1990s several new symbiotic stars with $V \lesssim 15$ mag have been found. This means that systematic searches for symbiotic stars may in principle reveal many new objects with, say, $V \lesssim 20$ mag. Any red giant with blue/ultraviolet excess may be a good candidate. However, spectroscopic observations are essential to confirm symbiotic nature of such candidates.

Acknowledgments. I gratefully acknowledge Maciej Mikołajewski and Toma Tomov for providing Figure 3. I would also like to thank the LOC for their support. This research was partly funded by KBN Research Grant No. 5 P03D 01920.

\section{References}

Allen, D.A. 1983, MNRAS, 204, 113

Anupama, G.C., Mikołajewska, J. 1999, A\&A, 344, 177

Belczyński, K., Mikołajewska, J., Munari, U., Ivison, R.J., Friedjung, M. 2000, A\&AS, 146, 407

Belyakina, T.S. 1979, Izv. KAO, 59, 133

Belyakina, T.S. 1984, Izv. KAO, 68, 108

Belyakina, T.S. 1991, Izv. KAO, 83, 118

Belyakina, T.S. 1992, Izv. KAO, 84, 45

Haniff, C.A., Scholtz, M., Tuthill, P.G. 1995, MNRAS, 276, 640

Hinkle, K.H., Fekel, F.C., Johnson, D.S., Scharlach, W.W.G. 1993, AJ, 105, 1074

Galis, R., Hric, L., Friedjung, M., Petrik, K. 1999, A\&A, 348, 533

Kenyon, S.J., Oliversen, N.A., Mikołajewska, J., Mikołajewski, M., Stencel, R.E., Garcia, M.R., Anderson, C.M. 1991, AJ, 101, 637

Kenyon, S.J., Mikolajewska, J., Mikolajewski, M., Polidan, R.S., Slovak, M.H. 1993, AJ, 106, 1573

Khudyakova, T.N. 1989, PhD Thesis, Leningrad University

Mattei, J.A. 1997, JAAVSO, 25, 57

Meinunger, L. 1981, MVS, 9, 67

Mikołajewska, J. (ed.) 1997, Physical Processes in Symbiotic Binaries and Related Systems, Copernicus Foundation for Polish Astronomy, Warsaw

Mikołajewska, J. 1999, in Stecklum, B., Guenther, E., Klose, S., eds, Optical and Infrared Spectroscopy of Circumstellar Matter, ASP Conf. Ser., vol. 188,291 
Mikołajewska, J., Ivison, R.J. 2001, MNRAS, 324, 1023

Mikołajewska, J., Kenyon, S.J. 1992, AJ, 103, 579

Mikolajewska, J., Brandi, E., Hack, W., Whitelock, P.A., Barba, R., Garcia, L., Marang, F. 1999, MNRAS, 305, 190

Mikołajewska, J., Brandi, Garcia, L., Ferrer, O., W., Whitelock, P.A., Marang, F. 2001, in Szczerba R. et al., eds, Post-AGB Objects as a Phase of Stellar Evolution, Kluwer, in press, astro-ph/0103495

Mikołajewski, M., Mikołajewska, J., Khudyakova, T.N. 1992, A\&A, 254, 127

Mikołajewski, M., Tomov, T., Mikołajewska, J. 1987, Ap\&SS, 131, 733

Mikołajewski, M., Mikołajewska, J., Tomov, T., Kulesza, B., Szczerba, R., Wikierski, B. 1990, Acta Astr., 40, 129

Munari, U., Yudin, B.F., Kholotilov, E., Tomov, T. 1996, A\&A, 311, 484

Whitelock, P.A. 1998, in Takeuri, M., Sasselov, D., eds, Pulsating Stars - Recent Developments in Theory and Observation, Universal Academy Press, Tokyo, 31 